\title{
Computational technologies in tissue engineering
}

\author{
H. A. Almeida \& P. J. Bártolo \\ Centre for Rapid and Sustainable Product Development of the \\ Polytechnic Institute of Leiria, Portugal
}

\begin{abstract}
In last decades, many advances have been made in order to aid the medical community. Numerous computational technologies have been developed and improved the efficiency in diagnostic and treatment of many diseases. Many of the technologies were developed with the main goal of aiding in the research of genetic and viral diseases. Tissue engineering is a multidisciplinary field that requires the combined effort of cell biologists, engineers, material scientists, mathematicians, geneticists, and clinicians toward the development of biological substitutes that restore, maintain, or improve tissue function. The success of this emerging medical domain relies on the current technological advances. This paper presents an overview of the existing computational technologies that have been implemented in tissue engineering and the design of scaffolds for tissue engineering applications. These computational technologies contemplate medical imaging processing, numerical calculations (structural, vascular and topological) and biofabrication techniques necessary for the scaffolds optimum design and production.
\end{abstract}

Keywords: tissue engineering, scaffolds, computational technologies, biofabrication, scaffold design.

\section{Introduction}

Tissue engineering is a multidisciplinary field that requires the combined effort of cell biologists, engineers, material scientists, mathematicians, geneticists, and clinicians toward the development of biological substitutes that restore, maintain, or improve tissue function [1]. Initially defined by Skalak and Fox [2] as "the application of principles and methods of engineering and life sciences' toward 
the fundamental understanding of structure-function relationships in normal and pathological mammalian tissues and the development of biological substitutes to restore, maintain, or improve tissue function" is a major component of regenerative medicine.

Most strategies in tissue engineering have focussed on using biomaterials as scaffolds to direct specific cell types to organise into three-dimensional structures and perform differentiated functions. The three most common strategies which have been adopted for the creation of new tissues are: Cellbased strategies, Growth-factor-based strategies and the Scaffold-based strategies. The Scaffold-based approach is the most widely used strategy for tissue engineering $[3,4]$. In this approach, living cells are obtained from a tissue harvest, from either the patient (autograft) or a different person (allograft), and cultured in vitro in a scaffold to manipulate cell functions and guiding new tissue formation, in order to obtain suitable biological constructs for transplantation [5]. Scaffolds are critical elements allowing cell attachment, proliferation and differentiation, delivering and retaining cells and growth factors, enabling the diffusion of cell nutrients and oxygen, and the establishment of an appropriate mechano-biological environment for the cells to secrete their own extracellular matrices in an organised way [6].

To achieve these goals, an ideal scaffold must satisfy several biological and mechanical requirements [7]. They must be biocompatible (the scaffold material must be non-toxic allowing cell attachment, proliferation and differentiation) and biodegradable (the scaffold material must degrade into non-toxic products) structures with controlled degradation rate (the degradation rate of the scaffold must be adjustable in order to match the rate of tissue regeneration). Scaffolds must also have an appropriate macro and microstructure of the pores and shape, highly interconnected pore structure and large surface area to allow high cell seeding, to promote neovascularisation. Additionally, they must also be able to carry biomolecular signals such as growth factors. Regarding the mechanical requirements, they must have appropriate strength and stiffness to withstand stresses in the host tissue environment, and adequate surface finishing guaranteeing a good biomechanical coupling between the scaffold, the cultured tissue and the host tissue. Porosity is a key design parameter to ensure an adequate mass flow of nutrients and oxygen to the inside of the construct and metabolic waste and byproducts to the outside, without compromising its mechanical performance. Scaffolds should also be easy sterilised by either exposure to high temperatures or immersing in a sterilisation agent, remaining unaffected by either of these processes.

Computational technologies play an important role in developing optimised scaffolds for tissue engineering applications, since the design, to the production, to the in vitro culture, ending with the implantation in the patient. This paper presents a global overview of the existing computational technologies that have been implemented in tissue engineering and in the design of scaffolds for tissue engineering applications. 


\section{Computational technologies}

Besides the listed biological and mechanical requirements, an optimum scaffold should also have functional and anatomical requirements, the gradient functionality of the organ or tissue, the external geometry and size of the scaffold should be the same as those of the natural tissue in order for the scaffold to fit and anchor onto the defected location [8]. With an appropriate external geometry and size and gradient functionality, a better fixation can be achieved by the scaffold, while facilitating better stress distribution at the interface between the surrounding tissue and the scaffold. Another aspect to be considered is the biodegradability of the scaffold. This is a key aspect to be considered due to the fact that the biodegradation rate and tissue regeneration rate may not coincide, resulting in an unstable scaffold and tissue structure. If the tissue has a more rapid growth compared to the degradation of the scaffold, then the tissue will have less space than the one necessary, if the scaffold has a more rapid degradation compared to the tissue growth, then the scaffold and tissue structure will become fragile compromising the end objective due to the possible mechanical and biological solicitations that it may have.

In the past decades, several tools have been developed to support the design of scaffolds for tissue engineering applications. Examples of these systems are for example: Computer Aided System for Tissue Scaffolds (CASTS) developed by Naing et al. [9] and Computer-Aided Tissue Engineering (CATE) developed by Sun et al. [10]. Computer-Aided Design of Scaffolds (CADS) [11] is a computational tool developed by the Centre for Rapid and Sustainable Product Development of the Polytechnic Institute of Leiria which will be described further on in this chapter. These computation tools integrate several tools ranging from design to engineering to manufacturing, all with the objective of aiding the design of scaffolds for tissue engineering applications. Figure 1 illustrates a simple concept of these systems. These computation tools integrate several tools ranging from design to engineering to manufacturing, all with the objective of aiding the design of scaffolds for tissue engineering applications.

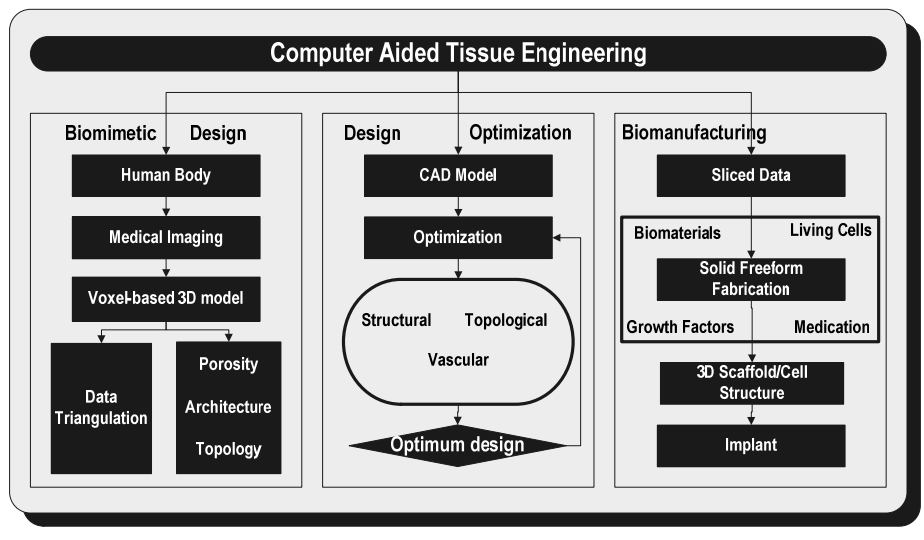

Figure 1: Computer aided tissue engineering. 
Finite element methods have also been used not only for the design of scaffolds, but also for the design of medical devices such as bioreactors for cell/scaffold cultures in Tissue Engineering applications [12]. The main objective is to design an optimal bioreactor system for optimal cell stimulation during the in vitro cell culture. Figure 2 illustrates the fluid velocity profile for a bioreactor without scaffold rotating about Y-axis (a), Z-axis (b) and biaxial rotation (c).

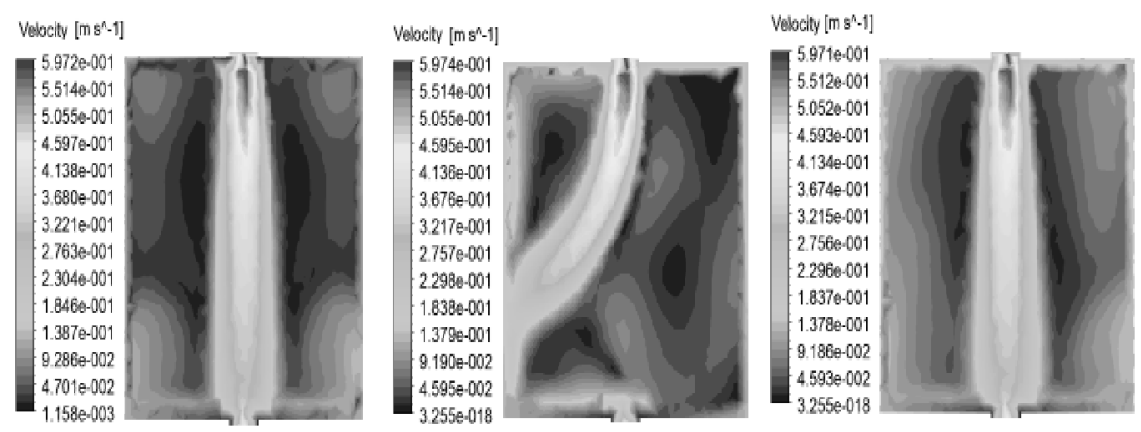

Figure 2: Velocity contour plots for the bioreactor without scaffold rotating about Y-axis (a), Z-axis (b) and biaxial rotation (c) [12].

\section{Scaffold modelling for tissue engineering}

The modelling of scaffolds for tissue engineering applications may be grouped into four main domains, as illustrated in figure 3:

- The analytical methods contain empirical relationships between structural parameters and mechanical properties. They provide only an estimate of the global mechanical properties.

- In the CAD-based modelling method, the porous structure is approximated using a 3D repetition of building blocks (called "unit cells”) with simply geometry. The unit cells can be generated by Boolean operations. Simple CAD software tools may be used in this process.

- $\quad$ The CT-based modelling uses an image-based approach where a 3D image of a structure serves as a basis for mesh generation.

- $\quad$ Several are the existing computational tools that may be used to process CT data and obtain 3D data of the desired anatomic model. Examples of these tools are Invesalius, Mimics, 3D-Doctor, Amira and Drishti.

- The Homogenisation theory is a multi-level approach where the microlevel model represents the exact micro-architecture of the structure, while the macro-level model is a continuum model of the whole structure. 


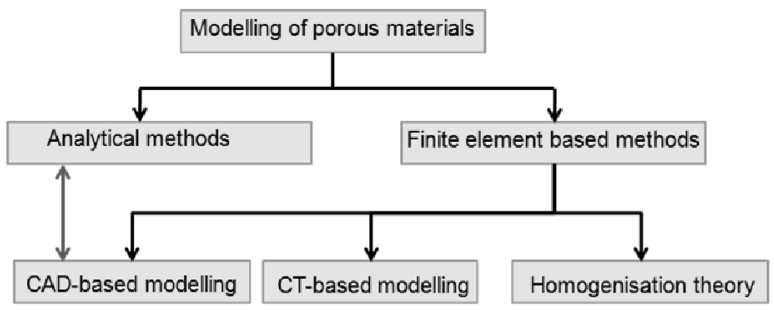

Figure 3: $\quad$ Modelling structure of CADS.

\section{Computer-Aided Design of Scaffolds (CADS)}

The prediction of the effective optimal properties of tissue scaffolds is very important for tissue engineering applications, either regarding mechanical, vascular or topological [13] properties. To aid this specific issue of the design process of scaffolds, a computational tool, Computer-Aided Design of Scaffolds (CADS) [11], has been developed which enables to quantify the structural heterogeneity and mechanical and vascular properties of a scaffold with a designed macro and microstructure [5]. Besides these topics, this computational tool already contemplates other issues such as degradation. In this case, it was possible to predict the mechanical properties of alginate scaffolds considering the influence of the water loss/shrinkage phenomenon [14].

Computer-Aided Design of Scaffolds (CADS) makes the bridge between different computer applications by allowing to material database tools (knowledge based systems for materials selection), CAD modelling systems, numerical simulation tools for structural, topological and fluid dynamics optimisation based on the Finite Element Method. Figure 4 illustrates 3 numerical simulations used in scaffold design: a) structural and b) vascular evaluation of the scaffold's performance, and c) a topological optimisation of a scaffold for a specific structural application.

a)

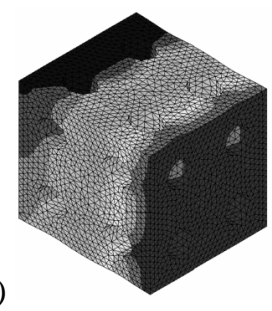

b)

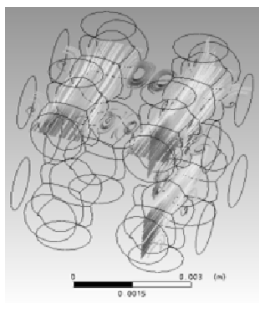

c)

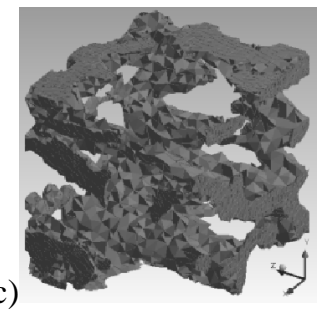

Figure 4: Examples of numerical simulations for scaffold design: a) structural simulations, b) vascular simulations, and c) topological optimisations.

Reviews of computer-aided design of scaffolds in tissue engineering can be found in "Structural and Vascular Analysis of Tissue Engineering Scaffolds: Part 1 - Numerical Fluid Analysis” [15], "Structural and Vascular Analysis of 
Tissue Engineering Scaffolds: Part 2 - Topology Optimization” [16] and "Computer-Aided Tissue Engineering” [17].

\section{Biomanufacturing technologies for tissue engineering}

As mentioned before, scaffolds must be processed into open porous structure that fills the tissue defect enabling tissue ingrowth. Additive technologies, also designated as biomanufacturing technologies, are considered to be the most viable method to fabricate scaffolds for tissue engineering applications. Biomanufacturing was initially defined in 2005 during the Biomanufacturing Workshop hosted by Tsinghua University in China as "the use of additive technologies, biodegradable and biocompatible materials, cells, growth factors, etc., to produce biological structures for tissue engineering applications” [18]. More recently, during the Spring 2008 Meeting sponsored by the American National Science Foundation, biomanufacturing was defined as "the design, fabrication, assembly and measurement of bio-elements into structures, devices, and systems, and their interfacing and integration into/with larger scale structures in vivo or in vitro environment such that heterogeneity, scalability and sustainability are possible”. In 2009, during the 59th General Assembly of the International Academy for Production Engineering (CIRP) in Boston it was established a collaborative working group on biomanufacturing that is based on three main pillars: a) Biofabrication, b) Biomechatronics and c) Biodesign and Assembly.

Figure 5 provides a general overview of the necessary steps to produce scaffolds for tissue engineering through biomanufacturing technologies [19]. The first step is the generation of the corresponding computer solid model through one of the currently available medical imaging techniques, such as computer tomography, magnetic resonance imaging, etc. These imaging methods produce continuous volumetric data (voxel-based data), which provide the input data for the digital model generation [19]. The model is then tessellated as an STL file (figure 6). The STL model is then mathematically sliced into thin layers (sliced model). This data is then sent to a biomanufacturing device in order to produce the biocompatible and biodegradable construct containing or not cells and growth factors. Once produced the scaffold model, it is then ready for implantation.

- Stereolithographic processes: Micro Stereolithography [21], Stereolithography [22] and multi-material micro Stereo-thermallithography [23].

Stereolithographic processes produce three-dimensional solid objects in a multi-layer procedure through the selective photo-initiated cure reaction of a polymer [23]. These processes usually employ two distinct methods of irradiation. The first method is the mask-based method in which an image is transferred to a liquid polymer by irradiating through a patterned mask. The irradiated part of the liquid polymer is then solidified. In the second method, a direct writing process using a focused UV beam produces polymer structures. 


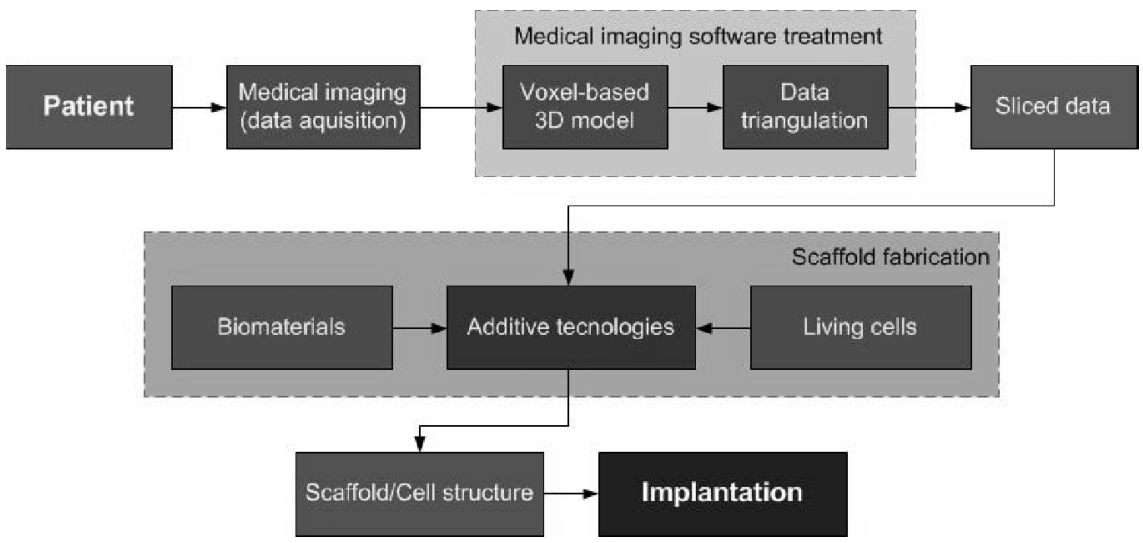

Figure 5: $\quad$ Steps of biomanufacturing in tissue engineering.

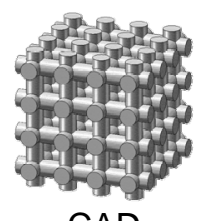

CAD

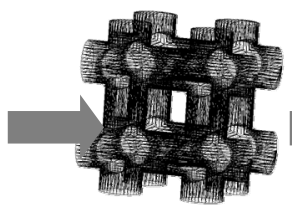

STL

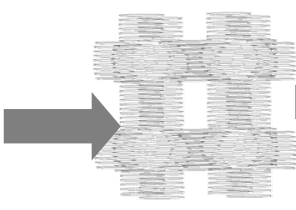

SLI

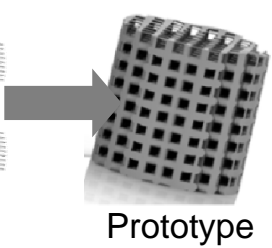

Figure 6: $\quad$ Flowchart of information to produce a 3D scaffold.

Biomanufacturing technologies are classified into four main fabrication processes such as [20]:

- $\quad$ Extrusion-based processes: 3D Fibre Deposition [24], Bioextruder [25], Bioplotting [26], Cell Printing [19], Direct Cell Writing [27], Fused Deposition Modelling [28], Precision Extruding Deposition [29], Pressure Assisted Microsyringe [30] and Rapid Prototyping Robotic Dispersing [31].

By this process, thin thermoplastic filaments are melted by heating and guided by a robotic device controlled by a computer, to form the object. The material leaves the extruder in a liquid form and hardens immediately. The previously formed layer, which is the substrate for the next layer, must be maintained at a temperature just below the solidification point of the thermoplastic material to assure good interlayer adhesion.

- $\quad$ Powder-bed fusion processes [32].

This process uses a laser emitting infrared radiation, to selectively heat powder material just beyond its melting point. The laser traces the shape of each cross-section of the model to be built, sintering powder in a thin layer. It also supplies energy that not only fuses neighbouring powder particles, but also bonds each new layer to those previously sintered. After each layer is 
solidified, the piston over the model retracts to a new position and a new layer of powder is supplied using a mechanical roller. The powder that remains unaffected by the laser acts as a natural support for the model and remains in place until the model is complete.

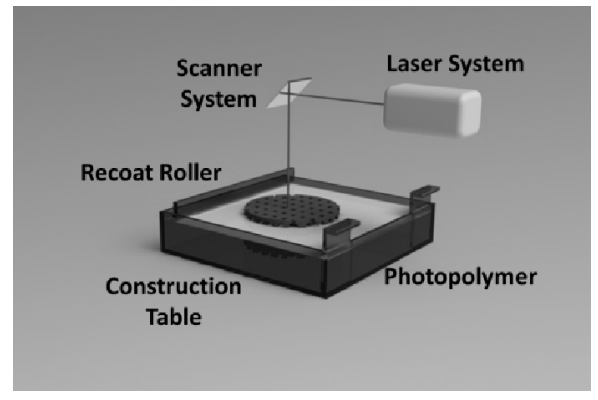

Figure 7: $\quad$ Illustration of the stereolithographic process.

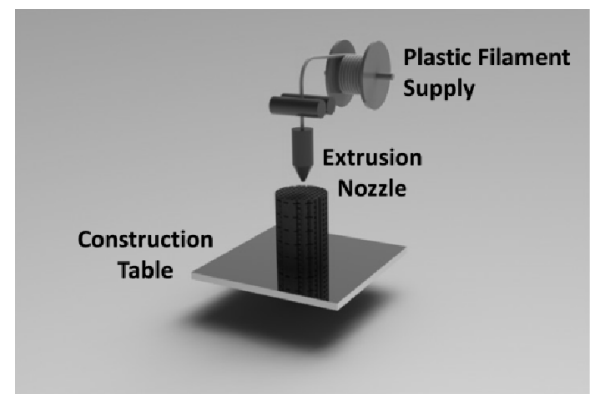

Figure 8: $\quad$ Illustration of the extrusion process.

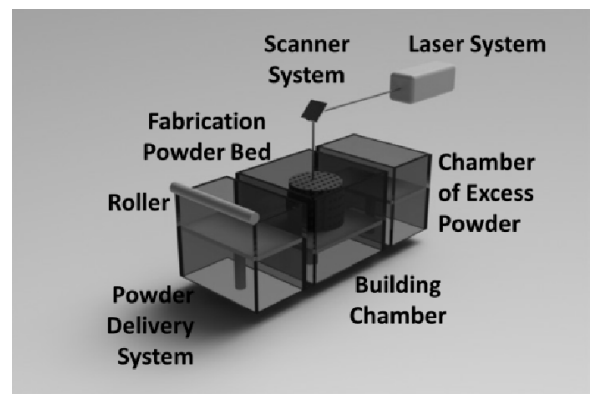

Figure 9: Illustration of the powder-bed fusion process. 
- $\quad$ Inkjet printing processes: 3D Printing [33] and ModelMaker II [34].

This process is divided into two main groups: the Binder Jetting and the Material Jetting process. a) the binder jetting process deposits a stream of particles of a binder material over the surface of a powder bed, joining particles together where the object is to be formed. After the fabrication the unbounded powder material is removed and the part is usually submitted to a sintering process. A piston lowers the powder bed so that a new layer of powder can be spread over the surface of the previous layer and then selectively joined to it. b) the material jetting techniques emit a stream of microparticles to an exact co-ordinate creating, layer-by-layer, the construct.

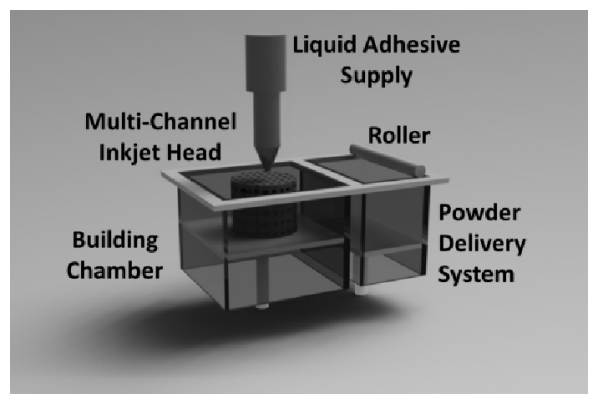

Figure 10: Illustration of inkjet printing processes; in this case, binder jetting.

The main advantages of biomanufacturing technologies are both the capacity to rapidly produce very complex 3D models and the ability to use various raw materials. In the field of tissue engineering, biomanufacturing technologies are used to produce scaffolds with customised external shape and predefined internal morphology, allowing good control of pore distribution and size [1].

Reviews of biomanufacturing technologies in tissue engineering can be found in "Biomedical production of implants by additive electro-chemical and physical processes” [35], "Biomanufacturing for Tissue Engineering: Present and Future Trends" [20], "Rapid Prototyping and Manufacturing for Tissue Engineering Scaffolds" [5], “Advanced Processes to Fabricate Scaffolds for Tissue Engineering" [1], "Virtual Prototyping and Bio Manufacturing in Medical Applications" [36] and "Bio-Materials and Prototyping Applications in Medicine” [37].

\section{Conclusions}

In last decades, many advances have been made in order to aid the medical community. Numerous computational technologies have been developed and improved the efficiency in diagnostic and treatment of many diseases. Many of the technologies were developed with the main goal of aiding in the research of genetic and viral diseases. Tissue engineering is a multidisciplinary field that requires the combined effort of cell biologists, engineers, material scientists, 
mathematicians, geneticists, and clinicians toward the development of biological substitutes that restore, maintain, or improve tissue function. The success of this emerging medical domain relies on the current technological advances. This chapter presents the state-of-the-art of computational technologies used in tissue engineering and the design of scaffolds for tissue engineering applications. These computation tools integrate several tools ranging from design to engineering to manufacturing, all with the objective of aiding the design of scaffolds for tissue engineering applications.

\section{Acknowledgements}

This research is supported by the Portuguese Foundation of Science and Technology through a PhD grant of Henrique Almeida (SFRH/BD/37604/2007). This research is also sponsored by the Portuguese Foundation for Science and Technology through a research project "Multimaterial microstereo-thermallithography ( $\mu$ STLG)” (PTDC/EME-PME/098037/2008) and through a Marie Curie Research Project designated "International Research Exchange for Biomedical Devices Design and Prototyping” “IREBID”.

\section{References}

[1] Bártolo, P.J., Almeida, H.A., Rezende, R.A., Laoui, T. and Bidanda, B. (2008) "Advanced Processes to Fabricate Scaffolds for Tissue Engineering”, in Virtual Prototyping and Bio-Manufacturing in Medical Applications, B. Bidanda and P.J. Bártolo (Eds.), Springer.

[2] Skalak, R. and Fox, C.F. (1988) “Tissue engineering”, Alan R. Liss, New York.

[3] Fuchs, J.R., Nasseri, B.A. and Vacanti, J.P. (2001) “Tissue engineering: a 21st century solution to surgical reconstruction”, Ann Thorac Surg, 72, 577-581, 2001.

[4] Liu, C.Z. and Czernuszka, J.T. (2006) "On the development of biodegradable scaffolds for tissue engineering: a perspective”, Materials Science and Technology, 12: 2479-2488.

[5] Bártolo, P.J., Almeida H. and Laoui, T. (2009) "Rapid prototyping and manufacturing for tissue engineering scaffolds”, International Journal of Computer Applications in Technology, 36(1): 1-9.

[6] Almeida, H.A. and Bártolo, P.J. (2010), "Virtual Topological Optimization of Scaffolds for Rapid Prototyping”, Medical Engineering and Physics, 32(7): 775-782.

[7] Tarawneh, A.M., Wettergreen, M. and Liebschner, M.A.K. (2012) "Computer-Aided Tissue Engineering: Benefiting from the Control Over Scaffold Micro-Architecture”, Computer-Aided Tissue Engineering, M. Liebschner (Ed.), Springer, 1-25.

[8] Leong, K.F., Chua, C.K., Sudarmadjia, N. and Yeong, W.Y. (2008) "Engineering functionally graded tissue engineering scaffolds", Journal of Mechanical Behaviour of Biomedical Materials, 1: 140-152. 
[9] Naing, M.W., Chua, C.K., Leong, K.F. and Wang, Y. (2005) "Fabrication of customised scaffolds using computer aided design and rapid prototyping techniques”, Rapid Prototyping Journal, 11: 249-259.

[10] Sun, W., Darling, A., Starly, B., and Nam, J. (2004) "Review - Computer aided tissue engineering: overview, scope and challenges”, Biotechnology Applied Biochemistry, 39:29-47.

[11] Almeida, H.A. and Bártolo, P.J., (2008) "Computer Simulation and Optimisation of Tissue Engineering Scaffolds: Mechanical and Vascular Behaviour", 9th Biennial ASME Conference on Engineering Systems Design and Analysis (ESDA2008).

[12] R.F.B. Pereira, D.M.F. Freitas, A.P.O. Tojeira, H.A. Almeida, N. Alves and P.J. Bártolo (2012), “Computational optimization of a novel bioreactor for tissue engineering applications”, Proceedings of the 1st International Conference on Design and Processes for Medical Devices (PROMED), E. Cerretti et al. (Eds.), Neos Edizioni, 217-221.

[13] Almeida, H.A, Bártolo, P.J. and Ferreira, J. (2007) "Design of Scaffolds Assisted by Computer”, in Modelling in Medicine and Biology VII, C.A. Brebbia (Ed.), Wit Press, 157-166.

[14] Almeida, H.A. Bártolo, P.J. and Mendes, A. (2009) "Mechanical Evaluation of Alginate Scaffolds Considering the Water Loss/Shrinkage Process”, ICTE 2009 International Conference on Tissue Engineering An ECCOMAS Thematic Conference, P.J. Bártolo et al. (Ed.), IST Press, 317323.

[15] Almeida, H.A and Bártolo, P.J. (2012), "Structural and Vascular Analysis of Tissue Engineering Scaffolds: Part 1 - Numerical Fluid Analysis”, Computer-Aided Tissue Engineering, M. Liebschner (Ed.), Springer, 183207.

[16] Almeida, H.A and Bártolo, P.J. (2012), "Structural and Vascular Analysis of Tissue Engineering Scaffolds: Part 2 - Topology Optimization”, Computer-Aided Tissue Engineering, M. Liebschner (Ed.), Springer, 209236.

[17] Liebschner, Michael A.K. (2012) “Computer-Aided Tissue Engineering”, Springer (ISBN 978-1-61779-764-4).

[18] Bártolo, P.J. and Chua, C.K. (2008) "Editorial: Celebrating the 70th Anniversary of Professor Yongnian Yan: A life dedicated to science and technology”, Virtual and Physical Prototyping, 3(4):189-191.

[19] Bártolo, P.J. (2006) "State of the art of solid freeform fabrication for soft and hard tissue engineering”, Design and Nature III: Comparing design in nature with science and engineering, C.A. Brebbia (Ed.), WIT Press.

[20] Bártolo, P.J., Chua, C.K., Almeida, H.A., Chou, S.M. and Lim, A.S.C (2009) "Biomanufacturing for tissue engineering: present and future trends”, Virtual and Physical Prototyping, 4: 203.

[21] Gaspar, J., Bártolo, P.J. and Duarte, F.M. (2008) "Cure and rheological analysis of reinforced resins for stereolithography”, Materials Science Forum, 587/588: 563. 
[22] Cooke, M.N., Fisher, J.P., Dean, D., Rimnac, C. and Mikos, A.G. (2002) "Use of stereolithography to manufacture critical-sized 3D biodegradable scaffolds for bone ingrowth”, Journal of Biomedical Materials Research, 64B: 65-69.

[23] Bártolo, P.J. and Mitchell, G. (2003) "Stereo-thermal-lithography”, Rapid Prototyping Journal, 9: 150-156.

[24] Woodfield, T.B.F., Malda, J., de Wijn, J., Péters, F., Riesle, J. and van Blitterswijk, C.A. (2004) "Design of porous scaffolds for cartilage tissue engineering using a three-dimensional fiber-deposition technique”, Biomaterials, 25: 4149-4161.

[25] Mateus, A.J., Almeida, H.A., Ferreira, N.M., Alves, N.M., Bártolo, P.J., Mota C. and Sousa, J.P. (2008) "Bioextrusion for tissue engineering applications, in Virtual and Rapid Manufacturing”, P.J. Bártolo et al. (Eds.), Taylor and Francis.

[26] Landers, R., Pfister, A., Hubner, U., John, H., Schmelzeisen, R. and Mulhaupt, R. (2002) "Fabrication of soft tissue engineering scaffolds by means of rapid prototyping techniques”, Journal of Materials Science 37: 3107-3116.

[27] Chang, R., Nam, J. and Sun, W. (2008) "Effects of Dispensing Pressure and Nozzle Diameter on Cell Survival from Solid Freeform Fabrication-based Direct Cell Writing”, Tissue Engineering, 14(1): 41-48.

[28] Hoque, M.E., Feng, W., Wong, Y.S., Hutmacher, D.W., Li, S., Huang, M.H., Vert, M. and Bártolo, P.J. (2008) "Scaffolds designed and fabricated with elastic biomaterials applying cad-cam technique”, Tissue Engineering, 14A: 907.

[29] Shor, L., Guceri, S., Wen, X., Gandhi, M. and Sun, W. (2007) "Fabrication of three-dimensional polycaprolactone/hydroxyapatite tissue scaffolds and osteoblast-scaffold interactions in vitro”, Biomaterials, 28(35): 5291-5297.

[30] Vozzi, G., Flaim, C., Ahluwalia, A. and Bhatia, S. (2003) "Fabrication of PLGA scaffolds using soft lithography and microsyringe deposition”, Biomaterials, 24: 2533-2540.

[31] Ang, T.H., Sultana, F.S.A., Hutmacher, D.W., Wong, Y.S., Fuh, J.Y.H., Mo, X.M., Loh, H.T., Burdet, E. and Teoh, S.H.(2002) "Fabrication of 3D chitosan-hydroxyapatite scaffolds using a robotic dispersing system”, Materials Science and Engineering, C20: 35-42.

[32] Tan, K.H., Chua, C.K., Leong, K.F., Cheah, C.M., Gui, W.S., Tan, W.S., Wiria, F.E.(2005) "Selective laser sintering of biocompatible polymers for applications in tissue engineering”, BioMedical Materials and Engineering 15: 113-124.

[33] Seitz, H., Rieder, W., Irsen, S., Leukers, B. and Tille, C. (2005) “Three dimensional printing of porous ceramic scaffolds for bone tissue engineering”, Journal of Biomedical Materials Research, 74B: 782-788.

[34] Yeong, W.Y., Chua, C.K., Leong, K.F., Chandrasekaran, M. and Lee, M.W. (2006) "Indirect fabrication of collagen scaffold based on inkjet printing technique”, Rapid Prototyping Journal, 12: 229-237. 
[35] Bártolo, P.J., Kruth, J.P., Silva, J., Levy, G., Malshe, A., Rajurkar, K., Mitsuishi, M., Ciurana, J. and Leu, M. (2012) "Biomedical production of implants by additive electro-chemical and physical processes”, CIRP Annals - Manufacturing Technology, 61(2): 635-655.

[36] Bidanda, B. and Bártolo, P.J. (2008) "Virtual Prototyping \& Bio Manufacturing in Medical Applications”, Springer (ISBN 978-0-38768831-2).

[37] Bártolo, P.J. and Bidanda, B. (2008) "Bio-Materials and Prototyping Applications in Medicine”, Springer (ISBN 978-0-387-47683-4). 